\title{
Governance to address health systems pitfalls of antibiotics overuse in low- and middle-income countries
}

\author{
Igor Francetic ${ }^{1,2,3}$ (D)
}

Received: 17 October 2018/Revised: 29 July 2019/Accepted: 24 August 2019/Published online: 29 August 2019

(C) Swiss School of Public Health (SSPH+) 2019

Over the last decades, health systems, including those in low- and middle-income countries (LMICs), experienced a sharp trend of increased use of antibiotics (Laxminarayan et al. 2013). The surge in antimicrobial drugs consumption in LMICs is a result of both patient factors like culture, lack of health education, awareness or income and health systems factors like increasing availability of drugs on public, private, legal and illegal markets, inefficient regulation of prescription practices and incentive schemes that favour over-prescription (Radyowijati and Haak 2003). Each problem exacerbates the other, forming a vicious feedback loop. Whilst health benefits and risks associated with overuse of antibiotics (like antimicrobial resistance) are well documented (Laxminarayan et al. 2013), we often fail to consider the role of the health system.

The vicious circle described above creates an excessive demand for antibiotics and other drugs that degrades the quality of health services and challenges health systems in LMICs from multiple perspectives. Health systems must allocate more resources for medicines, which undermines the sustainability of systems supported by meagre budgets or that rely massively on external sources. Central medical stores and supply chains are, by nature, exposed to various forms of corruption and must also cope with the challenge of serving health facilities that may be spread over vast rural regions with rough roads that disappear in rainy

This Editorial is part of the series "Young Researcher Editorial", a training project of the Swiss School of Public Health $(\mathrm{SSPH}+)$.

Igor Francetic

igor.francetic@unibas.ch

1 Swiss Tropical and Public Health Institute (Swiss TPH), Basel, Switzerland

2 University of Basel, Basel, Switzerland

3 University of Applied Sciences and Arts of Southern Switzerland (SUPSI), Manno, Switzerland seasons, resulting in increased frequency of stock-outs in essential and other medicines.

Prescribing drugs, including antibiotics, slowly became a necessary condition to leave patients satisfied after their visits at the facility, even if clinically inappropriate. Health providers have increased prescription rates, frequently violating clinical guidelines to please patients and thus encouraging patients to ask for drugs. Patient satisfaction is a key determinant of their healthcare-seeking behaviour (at government-managed health facilities) and their adoption of voluntary social health protection schemes like community-based health insurance. When providers fail to meet patients' expectations, e.g. when medicines are out of stock, patients may be pushed towards private and traditional health providers or pharmacies, which may increase their out-of-pocket expenses, generating impoverishment and inequality (Radyowijati and Haak 2003). In fact, the current state of social health protection in most LMICs puts the burden of prescription payments in private markets on the poor, in a very regressive way (Mills et al. 2012).

The private health sector serves a large and increasing share of population in LMICs, including the poor (Bhattacharyya et al. 2010). Increased demand for antibiotics and other drugs, combined with characteristic problems in health systems in LMIC like lack of governance, regulation and enforcement, has created a boom in private pharmacies and drug stores. These mostly unregulated providers sell all kinds of drugs, including prescription medicines and antibiotics (Shet et al. 2015). Trades usually happen over-the-counter and against out-of-pocket payments. Pharmacies and drug stores have become de facto healthcare providers for much of the population in many LMICs (Mackintosh et al. 2016). They diagnose and prescribe without proper authority or training (Miller and Goodman 2016). Chain pharmacies have also entered the market, without improving quality of services even though they may be more specialized and have more available (Miller et al. 2018). 
From a socioeconomic and a health systems perspective, these problems contribute to the vicious cycle of antibiotic overuse. Addressing the symptoms by, for example, strengthening supply chains of medicines and providing subsidies to the poor is one possible solution. Health promotion and patient education aimed at increasing health literacy are two components fundamental to addressing the root causes of the problem and reducing the excess demand for antibiotics. But it may be cheaper and more effective if we also strengthen health systems governance.

Governance could be improved in four ways: better regulation; better incentives; better training; and more accountability. To control private drug stores and pharmacies and stop the flow of counterfeit drugs, LMICs need more regulations that will make markets more transparent and give people access to quality services offered by skilled personnel at reasonable prices. Health providers and prescribers, whether operating in public, private or faithbased organizations, should be exposed to better incentives that would help to break the link between drug prescription and remuneration. This would ease pressure on health systems and medicine supply chains by reducing the prescription volume and expose patients to fewer out-ofpocket payments for prescriptions, especially in the private sector. Health providers and personnel in pharmacies and drug stores could improve their compliance with clinical guidelines and promote safe and effective treatment if they were better trained. If regulators across LMICs enforced existing rules, and we devised mechanisms that favoured community participation and social accountability, all actors would become more accountable, which would improve efficiency and reduce inequity in health care.

Funding The author's PhD research is funded by the Swiss Programme for Research on Global Issues for Development (r4d), a joint initiative of the Swiss Agency for Development and Cooperation (SDC) and the Swiss National Science Foundation (SNF) (Grant No. 160373).

\section{Compliance with ethical standards}

Conflict of interest The author declares that he has no conflict of interest.
Ethical approval This article does not contain any studies with human participants or animals performed by any of the authors.

Informed consent This article does not contain any studies with human participants that required informed consent.

\section{References}

Bhattacharyya O, Khor S, McGahan A, Dunne D, Daar AS, Singer PA (2010) Innovative health service delivery models in low and middle income countries-what can we learn from the private sector? Health Res Policy Syst 8(1):24. https://doi.org/10.1186/ 1478-4505-8-24

Laxminarayan R, Duse A, Wattal C, Zaidi AKM, Wertheim HFL, Sumpradit N, Vlieghe E, Hara GL, Gould IM, Goossens H, Greko C, So AD, Bigdeli M, Tomson G, Woodhouse W, Ombaka E, Peralta AQ, Qamar FN, Mir F, Kariuki S, Bhutta ZA, Coates A, Bergstrom R, Wright GD, Brown ED, Cars O (2013) Antibiotic resistance- the need for global solutions. Lancet Infect Dis 13(12):1057-1098. https://doi.org/10.1016/S14733099(13)70318-9

Mackintosh M, Channon A, Karan A, Selvaraj S, Cavagnero E, Zhao $\mathrm{H}$ (2016) What is the private sector? Understanding private provision in the health systems of low-income and middleincome countries. Lancet 388(10044):596-605. https://doi.org/ 10.1016/S0140-6736(16)00342-1

Miller R, Goodman C (2016) Performance of retail pharmacies in low- and middle-income Asian settings: a systematic review. Health Policy Plan 31(7):940-953. https://doi.org/10.1093/hea $\mathrm{pol} / \mathrm{czw} 007$

Miller R, Hutchinson E, Goodman C (2018) 'A smile is most important'. Why chains are not currently the answer to quality concerns in the Indian retail pharmacy sector. Soc Sci Med 212:9-16. https://doi.org/10.1016/j.socscimed.2018.07.001

Mills A, Ataguba JE, Akazili J, Borghi J, Garshong B, Makawia S, Mtei G, Harris B, Macha J, Meheus F, McIntyre D (2012) Equity in financing and use of health care in Ghana, South Africa, and Tanzania: implications for paths to universal coverage. Lancet 380(9837): 126-133. https://doi.org/10.1016/S0140-6736(12)60357-2

Radyowijati A, Haak H (2003) Improving antibiotic use in lowincome countries: an overview of evidence on determinants. Soc Sci Med 57(4):733-744. https://doi.org/10.1016/S02779536(02)00422-7

Shet A, Sundaresan S, Forsberg BC (2015) Pharmacy-based dispensing of antimicrobial agents without prescription in India: appropriateness and cost burden in the private sector. Antimicrob Resist Infect Control 4(1):55. https://doi.org/10.1186/s13756015-0098-8

Publisher's Note Springer Nature remains neutral with regard to jurisdictional claims in published maps and institutional affiliations. 